\title{
Study on Generalized Predictive Optimization Control Algorithm Based on Wavelet Transform
}

\author{
Xinli Deng ${ }^{1, a}$, Bing Guo ${ }^{1, b^{*}}$ \\ ${ }^{1}$ Chongqing Radio \& TV University, Chongqing 400052, China \\ aguodeng@tom.com, ${ }^{b^{*}}$ cqguobing@126.com
}

Keywords: wavelet transform, generalized predictive, control algorithm.

\begin{abstract}
In order to overcome the influences of model mismatch or sometimes non-linear variables and interference in generalized predictive control process, that cause a lag, being unstable and uncertain and make the predictive result not ideal, the paper presented a generalized predictive optimization control algorithm based on wavelet transformation. In the paper, we made the anatomy of localized nature and characteristics in translation, expansion and contraction for wavelet analysis, constructed the implicit generalized predictive control algorithm based on wavelet analysis, and derived a sort of generalized predictive optimization control algorithm. The experiment contrast simulations demonstrated that it could greatly improve predictive control quality. The study results show that the improved algorithm presented in this paper can be higher in predictive control precision, faster in predictive operating time, and it provides a new algorithm for reference.
\end{abstract}

\section{Introduction}

Predictive control is a model-based control, this model is called predictive model. Function prediction model is based on historical information and future object input predict its future output. Predictive control is a rolling optimization algorithm that determines the optimal future control action by a performance index. According to the general forecast model and control strategy is optimized in the future it is a finite length of time from the moment of the current carried. According to the prediction model, at each sampling time to optimize performance only relates to a result, not a global predictive control from the same moment of the future for a limited time, and the next moment, the same time period onward optimization, optimization performance indicators, but every time there is a performance optimization with respect to the time. Relative forms at different times to optimize performance is the same, but its absolute form, that is included in the time zone is different.

Thus, predictive control, optimization is not once offline, but repeatedly conducted on-line. Generalized Predictive Control as a new remote predictive control, set the advantages of multiple algorithms as one, with better performance, attention has been paid. There are a variety of correction algorithm, it can be divided into two kinds of explicit and implicit algorithms. Especially Implicit Generalized Predictive Control does not recognize the object model parameters, but identification strike the optimal control law parameters directly from the input / output data, thus avoiding the line solving the Diophantine (Diophantine) equation brought a large number of intermediate operations reduce the computational workload, saving time. However, the implicit generalized predictive control, 
predictive process model mismatch or sometimes change, non-linear and interference effects, leading to lag prediction signal, unstable, uncertain, so the result is not ideal.

Consider prediction algorithm presence of defects, and the wavelet transform is a time-frequency positioning tool, with good localization properties, easy to do local analysis; and because the wavelet translation, of breathing function, facilitate analysis of non-stationary signals. According to the principle of wavelet analysis, wavelet analysis thought the introduction of predictive control algorithm, an optimization algorithm Implicit Generalized Predictive Control based on wavelet transform, and the simulation results show the effectiveness and superiority of the algorithm proposed.

\section{Data acquisition and data processing}

By using the principle of fluid natural oscillation and detecting formed Kaman vortex frequency after vortex occurs, the vortex flowmeter can find the volume flow rate through the pipeline, and the basic relation expression is shown as in formula (1).

$$
f=s_{t} \cdot \frac{v}{d}, f \in L^{2}(R)
$$

In which, $\boldsymbol{v}(\mathrm{m} / \mathrm{s})$ is the mean velocity of fluid that is not affected by noise interference, $d(\mathrm{~m})$ is the effective diameter of the vortex occurring body, and $f$ is the Kaman vortex frequency. In the measuring range ( $\operatorname{Re}$ from $5 * 103$ to $1.5^{*} 105$ ), $S_{t}$ is a constant, and the volume flow of fluid flowing through the pipe is shown as in formula (2).

$$
Q=\frac{\pi D^{2} f d}{4 S_{t}} \cdot\left(1-1.25 \cdot \frac{d}{D}\right)
$$

In which, $Q$ denotes the volume flow rate $\left(\mathrm{m}^{3} / \mathrm{s}\right)$, and $D$ is the pipe diameter (m). The formula (2) is the measurement basis of vortex flowmeter. From formula (2), it can be seen that the key of determining flow is to measure vortex frequency $f$. But due to the impact of factors such as change in velocity pulse by the fluid turbulent state, pipeline random vibration in industry field measurement and so on, the output signal of vortex frequency transmitter is not an ideal sinusoidal signal, but mixed with noise signals (mainly white noise), and therefore it must carry on filtering processing[7, 8].

\section{Signal processing of wavelet denoising}

The output of vortex flowmeter theoretically is a sine wave[9]. Although it contained different noise components in the output signal of actual vortex flow, but under the condition of the signal not to be drowned out by the interference, the main energy is still focused on the frequency of useful sine signal[10]. Based on the above theory, it can perform wavelet transform on the actual vortex signal, draw the diagram of signal in frequency domain, find the highest peak of spectrum and the biggest point of spectrum width to length ratio, and thereby extrapolate the point of the frequency for the measurement of vortex flow value [11]. 
The wavelet transform and the traditional Fourier transform (STFT) (namely windowed Fourier transform) come down in one continuous line[12], but the Fourier transform (STFT) has the advantages of less. The formula (3) is the signal $\mathrm{f}(\mathrm{x})$ wavelet transform under the wavelet function $\Psi$.

$$
W_{\Psi} f(b, a)=|a|^{-\frac{1}{2}} \int_{-\infty}^{\infty} f(x) \overline{\Psi\left(\frac{x-b}{a}\right)} d x
$$

The scale parameter a and displacement parameter $b$ in the wavelet transform can also change at the same time. For the high-frequency spectrum information, the time interval can be relatively reduced (scale a reduced) so as to make the frequency precision be better, and for the low frequency spectrum information, the time interval can be relatively increased (scale a increased) so as to make frequency information be summarized, and that is to say that this is called time-frequency localization. Based on the above characteristics, the wavelet analysis of signal processing is more fine and flexible, and it is more in time and effectively to remove the noise.

The ideal sine signal is continuous, differentiable and smooth, and the emergence of the noise has randomness. It is almost everywhere singular, and when it makes multi-scale wavelet transform for the two signals, the wavelet spectrum owns different characteristic. Its wavelet spectrum of effective signal increases with the scale, the number of maximum (high frequency signal point) is more stable, and it has obvious inheritance characteristic. But the number of random noise spectrum maximum points reduced with the scale increases significantly, and the emergence moment of maximum point between adjacent scales has no inheritance characteristic.

From the view of functional analysis, because the wavelet operator $\Psi$ is integrable on real line of Lebesgue square, it has the formula (4).

$$
\int_{-\infty}^{\infty}|\psi(x)|^{2} d x<\infty
$$

It is also absolutely integrable, i.e.

$$
\int_{-\infty}^{\infty}|\psi(x)| d x<\infty
$$

The assumption that the signal $\mathrm{x}$ is absolutely integrable, then it has formula (6).

$$
\|\psi(x)\| \leq\|\psi\| \cdot\|x\|
$$

The white noise is the noise signal of expectation being as zero, and it is absolutely integrable obviously[11]. With the increasing of scale (even at smaller scales), the time interval of integral increases, and the norm $\|x\|$ of noise signal $x$ will tend to expected value zero. And from formula (6), it can be seen that its wavelet spectrum expectation $\|\Psi(\mathrm{x})\|$ will also tend to zero. Particularly important is that for wavelet spectrum of effective mutation signal in the signal increases with the 
scale (at smaller scales), the norm is instead increased, the polarity is more obvious, and it has stronger inheritance characteristic.

Let $\mathrm{n}(\mathrm{x})$ be the real and wide stationary white noise, and the variance is $\sigma^{2}$, then the expectations of wavelet transform $W_{n}(s, x)$ for white noise is shown as in formula (7).

$$
E\left(\left|W_{n}(s, x)\right|^{2}\right)=\frac{\|\Psi\|^{2} \cdot \sigma^{2}}{s}
$$

In which, $\Psi$ is the wavelet operator, $s$ denotes the scale of wavelet transform, and it can be seen that the attenuation of $\mathrm{E}\left(\left|W_{n}(s, x)\right|^{2}\right)$ is proportional to $1 / s$, namely along with the increasing of scale, the amplitude on average of white noise wavelet transform reduces. If $\mathrm{n}(\mathrm{x})$ is Gauss white noise, then in the scale s, the average density of the wavelet transform is shown as in formula (8).

$$
d_{s}=\frac{1}{s \pi}\left|\frac{\left\|\Psi^{(2)}\right\|}{2\left\|\Psi^{(1)}\right\|}+\frac{\left\|\Psi^{(1)}\right\|}{\|\Psi\|}\right|
$$

In which, $\Psi^{(1)}$ and $\Psi^{(2)}$ are respectively the one-order and two-order derivative. From formula (8), it can be seen that $d_{s}$ is proportional to $1 / s$, and if the scale increases then the density decreases.

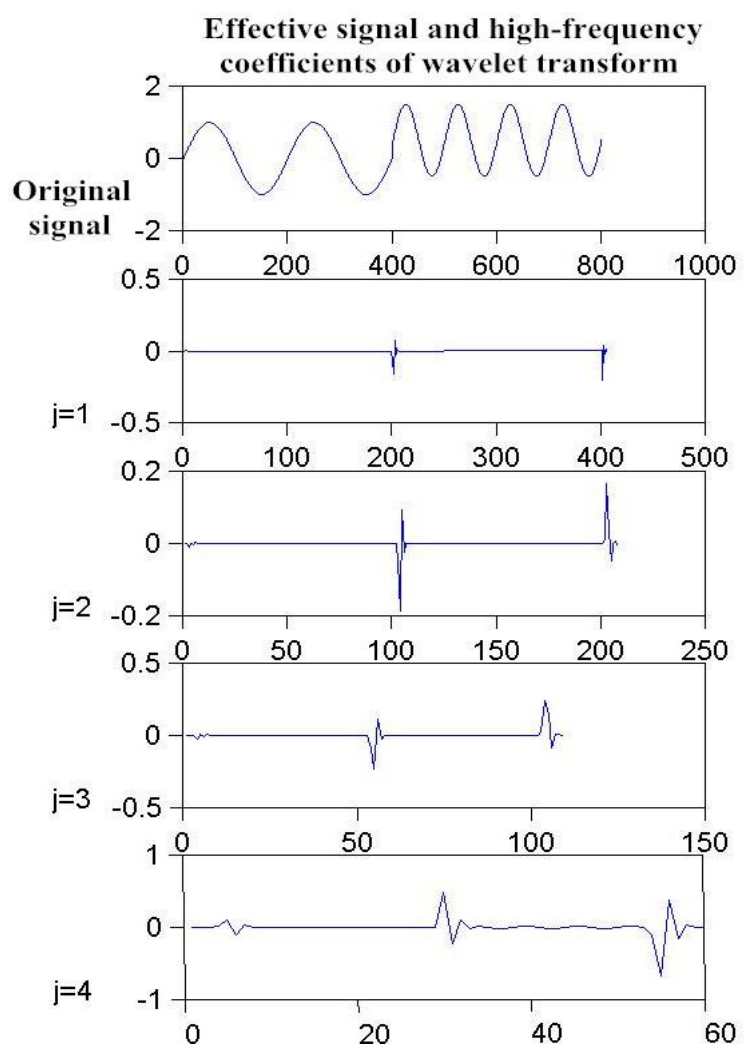

(a) Effective signal

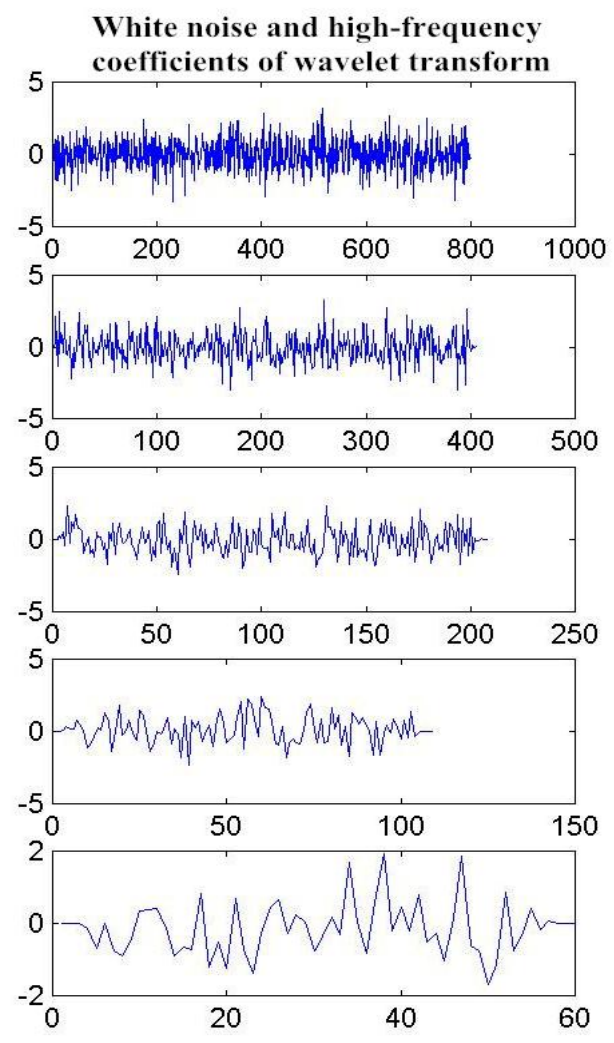

(b) Signal with noise

Fig.1 High frequency coefficients of wavelet transform

From the contrastive analysis for an effective signal and white noise and their high frequency coefficient of wavelet transform, it can get the results shown in Fig.1. The graphic in Fig.1 (a) and 
Fig.1 (b) is respectively the high frequency coefficient map of sine signal and white noise under condition of four scales db6 of wavelet transform. From Fig. 1(a), it can be seen that the original signal is composed of two sections of different frequency sine signal formed by splicing, and there are three mutation points. After making wavelet transform of four scales, the position of mutation signal point is highlighted, and with the increase of the scale of $j$, the number of extreme points is very stable. And the emergence of the approximate position is respectively as the following, if $\mathrm{j}=1$ then it is 0 , 200 and 400 , if $j=2$ then it is 0,100 and 200 , if $j=3$ then it is 0,50 and 100 , if $j=4$ then it is 0,25 and 50. The position of emergence is $1 / 2$ of maximum position number of previous scale, and it can be seen that it has very strong inheritance characteristic. From Fig. 1(b), it can be seen that the number and amplitude of extremum point of white noise wavelet spectrum decreases rapidly with increasing scale of $\mathrm{j}$, and the emergence location is any relation with scale change.

According to the above theory, as long as it removes the wavelet spectrum component generated by the noise on each scale, especially the noise wavelet spectrum component that the noise spectrum is dominated by scales, then what the wavelet spectrum preserved basically is the wavelet spectrum of effective signal. If it reuses the wavelet transform and reconstruction algorithm, then it can reconstruct the effective signal. The denoising process is as follows.

(1) Using Mallat algorithm [3], it can find the wavelet decomposition of noise signal $\mathrm{f}(\mathrm{x})$ in each scale $\mathrm{j}(\mathrm{j}<\mathrm{N})$.

$$
\begin{gathered}
S_{2^{j}} f(x)=\sum_{l \in z} h_{l} S_{2^{j-1}} f\left(x-2^{j-1} l\right) \\
W_{2^{j}} f(x)=\sum_{l \in z} g_{l} S_{2^{j-1}} f\left(x-2^{j-1} l\right)
\end{gathered}
$$

In which, $h, g$ denotes respectively the corresponding low-pass filter and high pass filter coefficient of the wavelet function $\Psi$. The formula (9) is the smooth signal of wavelet decomposition, and the formula (10) is its high frequency signal.

(2) Selecting threshold makes the soft threshold processing for high frequency coefficient from scale $\mathrm{N}$ to scale 1 . If some modulus maximum points of wavelet transform amplitude decrease with the scale increase sharply, then it shows that these maximum points are almost controlled by white noise, and it should be removed. If some modulus maximum points of wavelet transform do not spread to larger scales, then it should also be removed. Generally, it sets a threshold for high frequency coefficient in the high scale to filter out the noise wavelet spectrum, keeps the wavelet spectrum of effective mutation signal, and then finds out the wavelet spectrum of effective mutation signal for low scale. The theory proposed in the literature can be found in Mallat algorithm [4].

(3) From the low frequency coefficient of scale $\mathrm{N}$ (smooth signal) and high frequency coefficient through processing for from scale 1 to scale $\mathrm{N}$, it can makes the reconstruction of the signal so as to get the denoising signal. 
Many scholars have also put forward some wavelet denoising algorithms, for example, the iterative projection method between convex multiple Hilbert space (POCS) in reference [2], and simplified algorithm in reference [5] etc.

\section{Simulation result and its analysis}

In the experiment, the signal with noise is still composed of two sine signals of different frequency after stitching and mixing white noise.

The figure 2 (a) is the noise free signal, and figure 2 (b) is the signal with noise. It is visible, because of low in SNR, effective signal submerged in noise, and peak pulse has been replaced by the noise, and the vortex flowmeter is unable to correctly measure the flow.

Now it takes the db6 wavelet respectively to actualize the wavelet denoising of largest scale $\mathrm{N}$ from 3 to 8 for the signal with noise, and the simulation results are shown in Fig. 2.
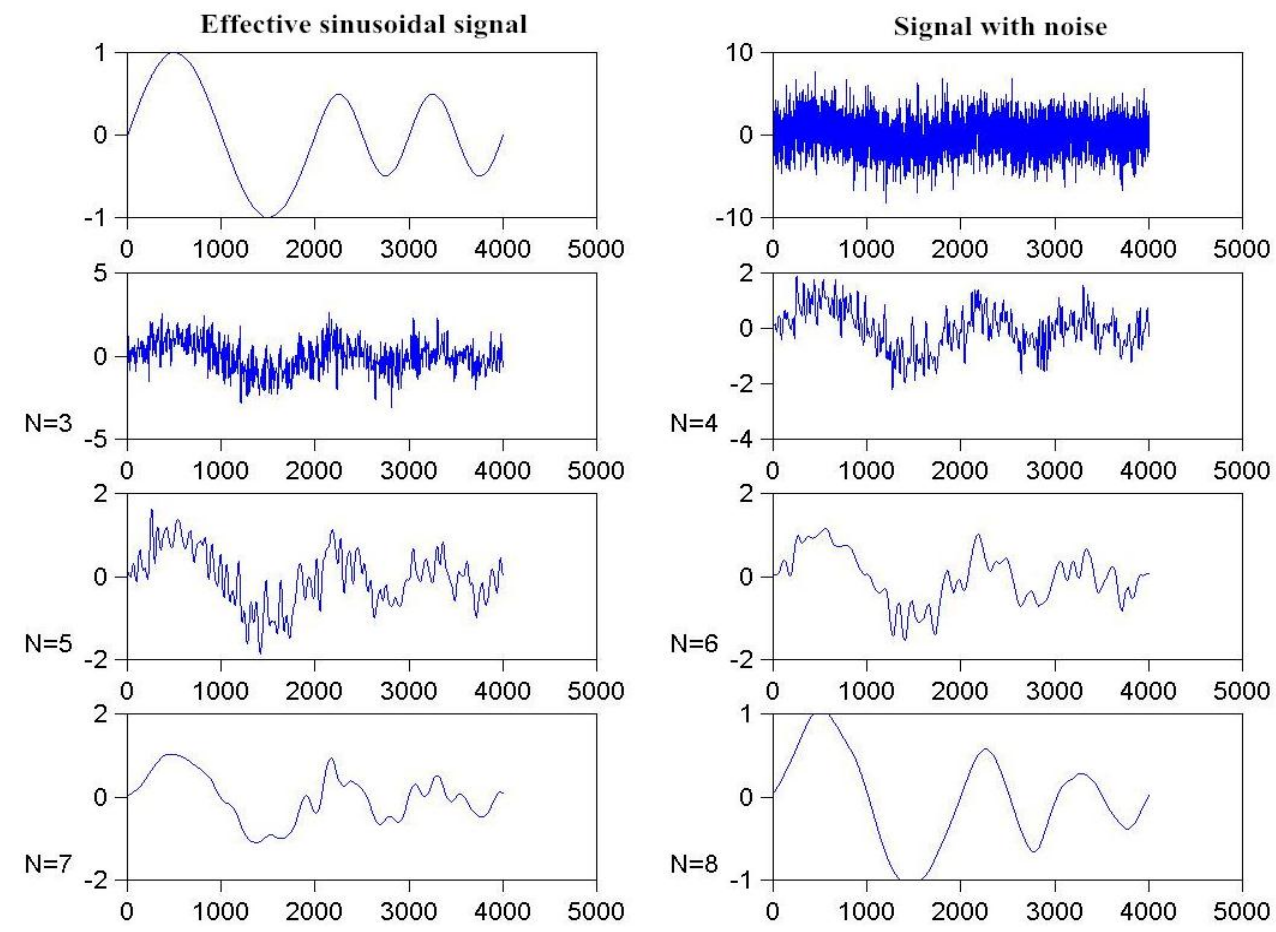

Fig. 2 Effect diagram of wavelet denoising simulation under each scale

From Fig. 2, it can be seen that with the increase of the scale of $\mathrm{N}$ the effect of noise filtering gradually becomes better. When it is the scale of $\mathrm{N}=8$ (the last figure), although the amplitude and the effective signal has also some differences, but the peak has come out prominently, and therefore the correct fluid frequency can be determined. Here what the scale needs to reach 8 is due to the SNR being small. It can be seen that for signals with different signal to noise ratio the selection of wavelet scale is different, and therefore it should be determined according to the effect. After considering the above some basic problems, it can actualize the signal processing for actual output signal of a stress type vortex flowmeter, and when the flow of vortex flowmeter is approximately to be as $0.50,0.60$, $0.70,0.80,0.90$ and $1.00 \mathrm{~m} / \mathrm{s}$, the result of signal acquisition and processing is shown as in Fig. 3 . 


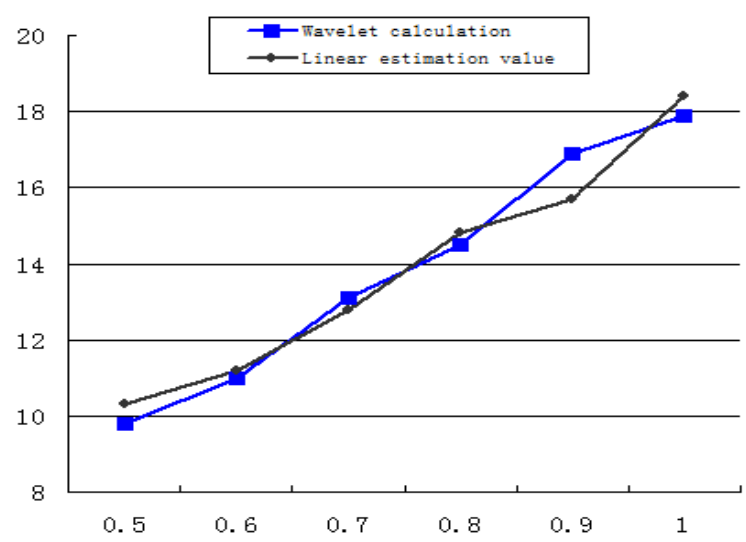

Fig.3 Approximate numerical analysis of periodic wavelet

Transverse coordinate in Fig.3 is the flow velocity for each sampling point, and the unit is $\mathrm{m} / \mathrm{s}$. The ordinate represents the average value of the periodic wavelet calculation. Through the analysis of power spectrum for the signal, it can find out the following conclusions.

(1) The average value of the periodic wavelet at different frequency calculated decreases with decreasing flow rate, and there is a linear relation.

(2) Using linear prediction method, it can get the standard output of vortex signal for each point above, calculate the analysis error spectrum, and all the error for each the point is less than $6 \%$

So, the Wavelet calculation has certain superiority in measuring low flow rate, but when the vortex flowmeter is lower flow rate (flow rate $<0.5 \mathrm{~m} / \mathrm{s}$ ), it is difficult to extract the useful signal by using wavelet analysis method. This is caused by defects of wavelet analysis method itself.

\section{Conclusions}

Observation of the three artificial picture, predictive control optimized and non-optimized predictive control compared to the former law optimal amount of change in the forecasting process smaller, reaching only change; the former results closer to the actual value, the faster the closer the actual value, especially in dual-input dual-output simulation, the better.

Simulation results show that the proposed method improves the accuracy of predictive control, fast, accurate forecasting, predictive control quality greatly improved.

Through the above analysis, the implicit generalized predictive control improved, while maintaining the original control algorithm stability and robustness, and can better predict the actual value, the result is closer to the true value.

\section{References}

[1] Al Asmi K and Castro I 1992 Vortex shedding in oscillatory flow: geometrical effects Flow Meas. Instrum. 3 187-202. 
[2] Amadi-Echendu, J.E.: Analysis of Signals from Vortex Flowmeter. Flow Meas. Flow Meas. Instrum. 4(4), 225-321 (1993).

[3] Baker R C 2000 Flow Measurement Handbook: Industrial Designs, Operating Principles, Performance, and Applications (Cambridge, UK: Cambridge University Press) .

[4] Venugopal A, Agrawal A and Prabhu S V 2010 Influence of blockage and upstream disturbances on the performance of a vortex flowmeter with a trapezoidal bluff body Measurement 43 603-16.

[5] Hebrard P, Malard L and Strzelecki A 1992 Experimental study of a vortex flowmeter in pulsatile flow conditions Flow Meas. Instrum. 3 173-86.

[6] Hu C C, Miau J J and Chou J H 2002 Instantaneous vortex-shedding behaviour in periodically varying flow Proc. R. Soc. A 458 911-32.

[7] Blodgett L 1992 Theoretical and practical design of pulsation damping systems Flow Meas. Instrum. 3 203-8.

[8] Rossberg A G, Riegler P, Buhl F, Herwig J and Timmer J 2004b Detection of improper installation from the sensor signal of vortex flowmeters Flow Meas. Instrum. 15 29-35.

[9] Rossberg A G, Bartholomé K and Timmer J 2004a Data-driven optimal filtering for phase and frequency of noisy oscillations: application to vortex flow metering Phys. Rev. E 69016216.

[10] H. J. Sun, T. Zhang and H. X.Wang, "Wavelet denoising method used in the vertex flowmeter," in Proceedings of 2nd international conference on machine learning and cybernetics, Xi'an, 2003, pp. 1109-1112. [11] F Laurantzon, A Segalini and P H Alfredsson,Time-resolved measurements with a vortex flowmeter in a pulsating turbulent flow using wavelet analysis,Measurement Science and Technology, Volume 21(12), 2010. [12] Miau J J, Hu C C and Chou J H 2000 Response of a vortex flowmeter to impulsive vibrations Flow Meas. Instrum. 11 41-9. 